\title{
Orbicularis oculi reflex in the Wallenberg syndrome: alteration of the late reflex by lesions of the spinal tract and nucleus of the trigeminal nerve
}

\author{
JUN KIMURA AND LYNN W. LYON \\ From the Department of Medicine (Neurology), University of Manitoba, \\ and the Department of EEG, Winnipeg General Hospital, Winnipeg, Canada, \\ and from the Department of Neurology, University Hospital, Iowa City, Iowa, U.S.A.
}

SUMMARY The orbicularis oculi reflex was studied in nine cases with lateral medullary lesions. Diagnosis of the Wallenberg syndrome was made clinically in seven cases and at necropsy in $\vec{\omega}$ another. The clinical features of one other case were closely allied to but not typical of this syn- $\stackrel{\rho}{S}$ drome. An afferent delay of the late reflex on the side of the lesion in the presence of a normal early reflex was seen in all but two cases. In one of the latter, the late reflex was normal and in the other, $\dot{\omega}$ a comatose patient, the late reflex was totally absent. It was concluded that the neurones of the first order responsible for the bilateral late reflex on unilateral stimulation terminate in the ipsilateral $i$ spinal nucleus of the trigeminal nerve without significant crossing over to the same structure on the $\infty$ other side. An afferent delay of the late reflex in the presence of a normal or nearly normal early reflex is consistent with a lateral medullary lesion implicating the spinal tract and nucleus. The Wallenberg syndrome is a common clinical entity showing this abnormality of the orbicularis ocudio reflex.

The response of the orbicularis oculi muscle to a tap over the brow (Kugelberg, 1952) or to an electrical stimulation of the supraorbital nerve (Rushworth, 1962a, b) has two separate components-an early ipsilateral reflex and a late bilateral reflex. It is generally agreed that the early reflex is transmitted through a simple arc, although whether it is myotatic (Kugelberg, 1952) or cutaneous (Tokunaga, Oka, Murao, Yokoi, Okumura, Hirata, Miyashita, and Yoshitatsu, 1958; Shahani, 1970) in nature is still in dispute. A delay of the early reflex is relatively specific to pontine lesions (Kimura, 1970; Namerow and Etemadi, 1970) provided the trigeminal (Kimura, Rodnitzky, and Van Allen, 1970) and facial nerves (Bender, Maynard, and Hastings, 1969; Kimura, Powers, and Van Allen, 1969; Kimura, 1971) are intact. The central pathway of the late reflex is less well understood. Kugelberg (1952) recognized a raised threshold of glabellar taps for the late reflex in four patients with trigeminal tractotomy and concluded that at least some part of this reflex passes over the spinal tract of the trigeminal nerve. Tokunaga et al. (1958) in their experimental study in cats, briefly mentioned that the threshold of the late reflex was raised after tractotomy. Alterations of the late reflex were also noted in a few patients with multiple sclerosis showing advanced bulbar signs (Kimura, 1970).

The purpose of this work has been to study the effect of lateral medullary lesions on the orbicularis oculi reflex. In the Wallenberg syndrome (Fisher, Karnes, and Kubik, 1961 ; Wilkins and Brody, 1970) infarction typically? involves, among other structures, the spinal tract and nucleus of the trigeminal nerve. With its clearly definable clinical features and well- 3 established pathological findings, this syndrome 0 serves as a good model for correlating lateral medullary lesions with alterations in the late을 reflex.

\section{METHOD AND NORMAL VALUES}

The technique used in this study for recording the orbicularis oculi reflex and the normal ranges of $<$ latency (mean value \pm 3 standard deviations) have? been described (Kimura et al., 1969). Recording@ simultaneously from surface electrodes placed over 


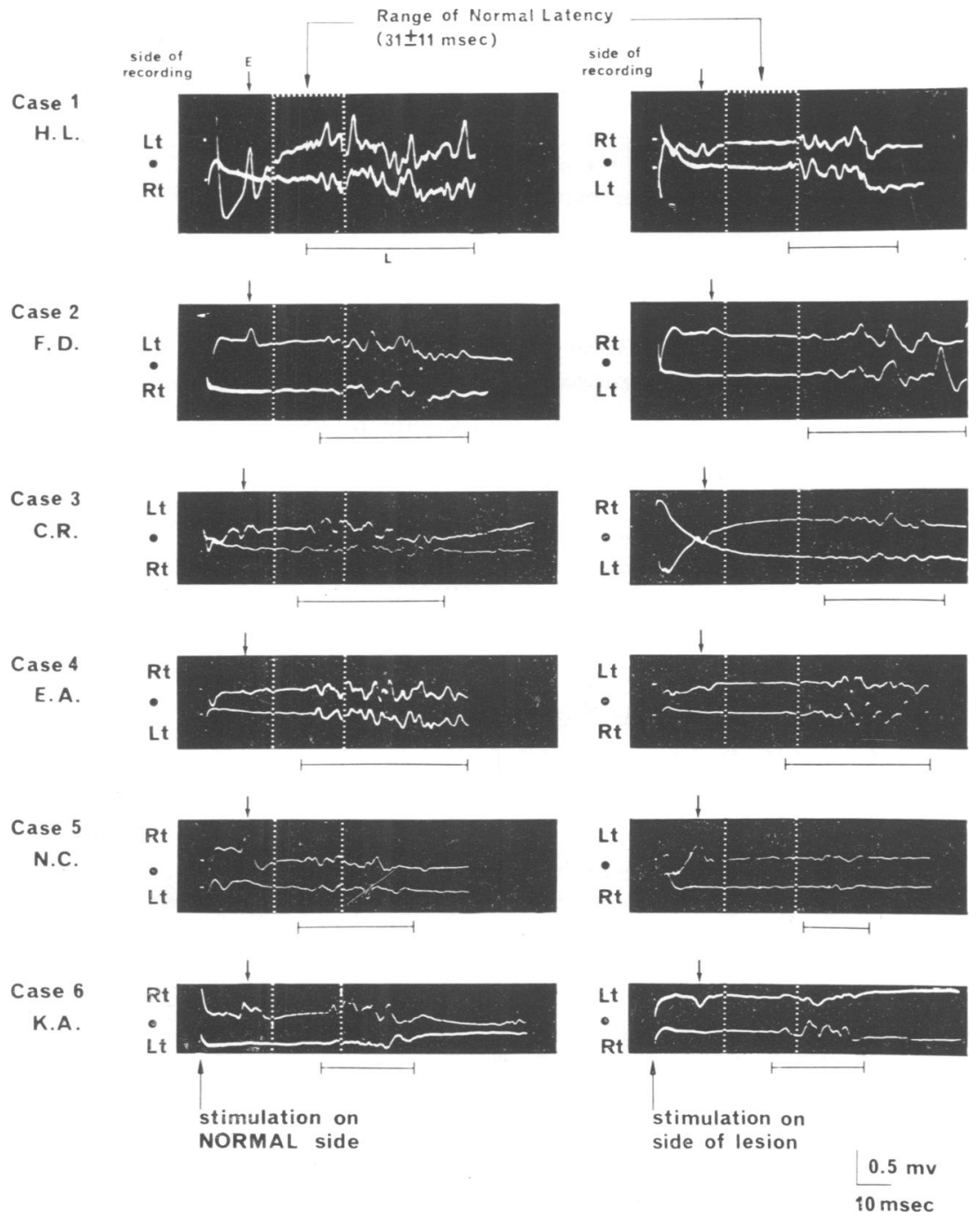

FIG. 1. Cases 1-6. Alteration of late reflex-lateral modullary syndrome. The stimulus was delivered to the supraorbital nerve and the reflex responses were simultaneously recorded from ipsilateral (upper tracing in each frame) and the contralateral (lower tracing in each frame) orbicularis oculi. Note a unilateral early reflex (small arrows) recorded only in the upper tracing and bilateral late reflexes (horizontal bars) recorded in both the upper and lower tracings in each frame. The late reflexes in the upper and lower tracings were equally delayed when the side of the lesion was stimulated (right half of the Figure) and normal when the normal side was stimulated (left half of the Figure) in cases 1 to 5 . In case 6, the late reflex was normal regardless of the side of stimulation. The dotted line indicates normal range of latency for the late reflexes on the basis of 30 normal subjects age 7 to 67 years (Kimura et al., 1969). The early reflex was normal in all. $E=$ early reflex. $L=$ bilateral late reflex. 
the right and the left orbicularis oculi muscles, unilateral stimulation of the supraorbital nerve evokes the early reflex (latency: $10 \cdot 6 \pm 2.5 \mathrm{msec}$ ) on the side ipsilateral to the shock and the late reflex on both sides. It is convenient to consider the latter in analogy with the pupillary light reflex and refer to the ipsilateral component as the direct late reflex (latency: $31 \pm 10 \mathrm{msec}$ ) and to the contralateral component as the consensual late reflex (latency: $32 \pm 11 \mathrm{msec}$ ). Thus, stimulation on the right, for example, evokes a right early reflex, a right direct late reflex, and a left consensual late reflex. (The consensual late reflex is named after the side of recording and not after the side of stimulation).

In addition to the criterion of normal range of latencies as mentioned above, normal range of variation between the two sides of one individual has also been established. For the early reflex a latency difference between the right and left sides exceeding $1.2 \mathrm{msec}$ is considered abnormal (Kimura et al., 1969.)

For the late reflex, two types of comparison are possible. Using simultaneous recordings from both sides of the face but stimulating only the right side, for example, permits one to make an instantaneous comparison between the right direct and left consensual late reflexes. Latency difference exceeding $5 \mathrm{msec}$ (Kimura et al., 1969) is considered abnormal. A unilateral delay of the late reflex on one side (regardless of the side of stimulation) implicates the efferent (final common) path on the same side in analogy with the pupillary light reflex in oculomotor palsy.

Secondly, bilateral late reflexes evoked by rightsided stimulation may be compared with those subsequently evoked by left-sided stimulation. This comparison, which requires separate stimulation on each side, is meaningful only when a shock of the same intensity is delivered to either side by the use of a constant current unit. The intensity of the shock is determined by slowly advancing the current to a level above which the evoked potential remains constant. It is also important to obtain several responses to document a consistent difference between the responses to right-sided, and to left-sided stimulation. If these precautions are taken, a latency difference exceeding $8 \mathrm{msec}$ (Kimura et al., 1970) is considered abnormal. A bilateral delay of the late reflexes on right-sided stimulation, for example, implicates the afferent (initial common) path on the right in analogy with the pupillary light reflex in right optic atrophy.

\section{RESULTS}

In seven out of nine patients (cases 1 to 7 ) included in this study, the clinical features were typical of the Wallenberg syndrome as shown in the Table. Case 8 was admitted in coma and the diagnosis was made at necropsy. In case 9 the $\bar{z}$ diagnosis of the Wallenberg syndrome was not $\stackrel{\mathbb{}}{=}$ justified, as the only unequivocal clinical finding was that of hemifacial hypalgesia and slight hypaesthesia associated with nausea and vomit- 0 ing. In five patients (cases 1,2, 3, 8, and 9) the lesions were on the right and in the rest (cases $4 \frac{3}{2}$ to 7) on the left.

The orbicularis oculi reflex was abnormal and showed essentially the same selective alteration $\stackrel{\overrightarrow{\vec{S}}}{\rightarrow}$ of the late reflex in six out of the seven clinically typical cases of the Wallenberg syndrome. These $\frac{\bar{\sigma}}{\bar{\omega}}$ are shown in Fig. 1 (cases 1 to 5) and Fig. 2 (case $\frac{\omega}{\sigma}$ 7). The findings were characterized by a bi- $\propto$ lateral delay (cases 4 to 5) or absence (case 7) of the late reflexes on left-sided stimulation when $\overrightarrow{0}$ the lesion was on the left and a bilateral delay $\overrightarrow{\vec{\omega}}$ (cases 1 to 3 ) of the late reflexes on right-sided $\stackrel{\omega}{\omega}$ stimulation when the lesion was on the right. The early reflex on the side of the lesion, although still within normal limits, was slightly longer in o latency when compared with the normal side in three patients (cases 2, 3, and 7). In the others $\underset{\infty}{N}$

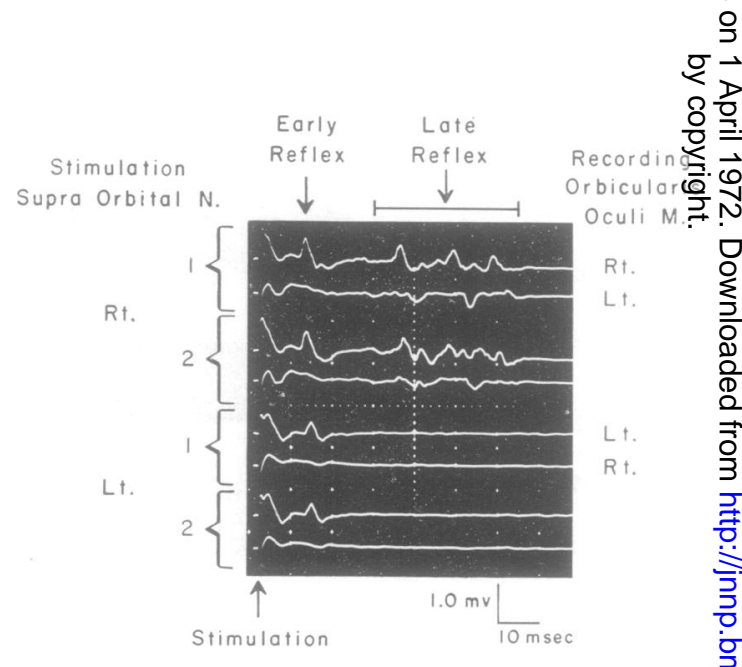

FIG. 2. Case 7. Left lateral medullary syndrome. Two successive stimulations (to show consistency) $ᄋ$ separated by a minimum of 15 seconds on the right $₹$ (top two pairs) followed by two successive stimulations 은 on the left with the same interval (bottom two pairs). $>$ The reflex responses were simultaneously recorded 으․ from ipsilateral (upper tracing in each pair) and the contralateral (lower tracing in each pair) orbicularis o oculi. Note a unilateral early reflex recorded only in $N$ the upper tracing and bilateral late reflexes recorded $N_{\mathrm{W}}$ in both the upper and lower tracings in each pair. The late reflexes were consistently absent bilaterally when the side of the lesion (left) was stimulated and normal $\stackrel{\odot}{\varnothing}$ bilaterally when the normal side (right) was stimulated. @ The early reflex was normal on either side. 
TABLE

ORBICULARIS OCULI REFLEX IN NINE PATIENTS WITH LATERAL MEDULLARY LESIONS

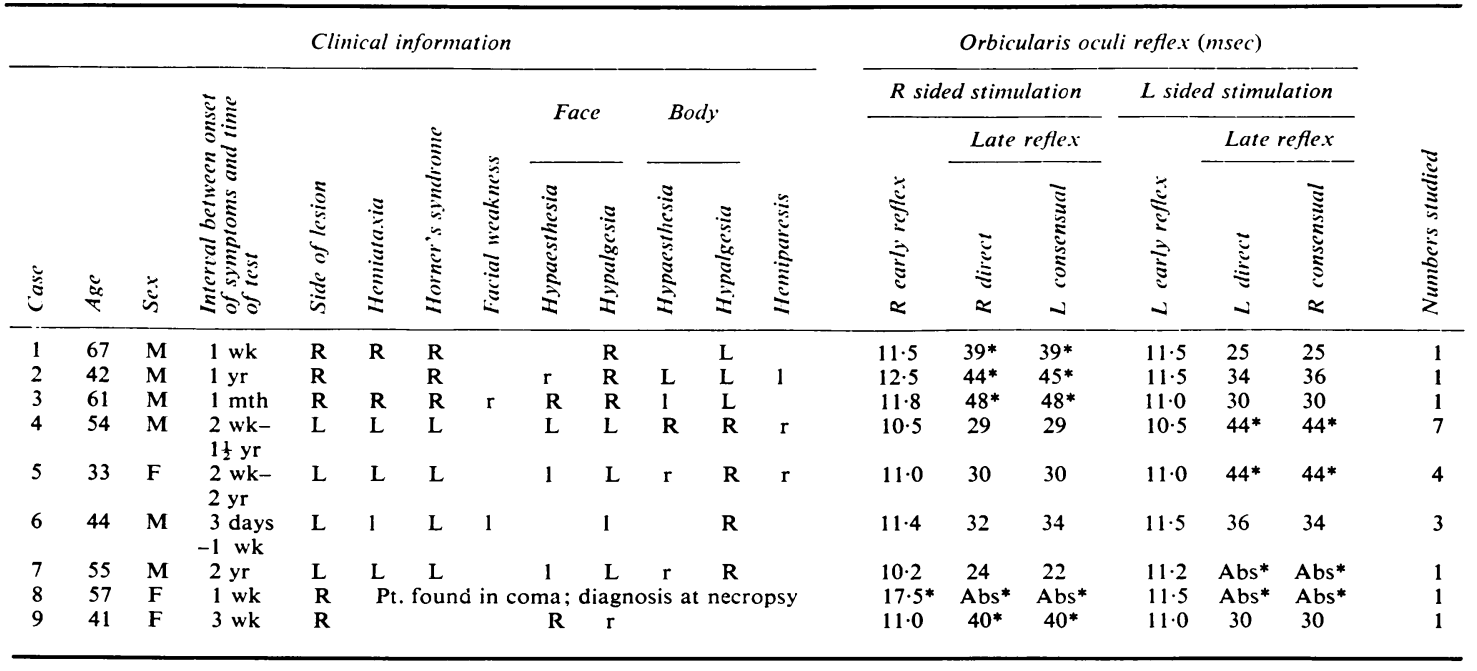

R, L moderate to considerable imvolvement on right or left sides.

$r, 1$ minimal to slight involvement on right or left sides. abnormal response.

(cases 1,4 , and 5) it was normal, showing no latency difference between the two sides. Two of these patients (cases 4 and 5) were tested serially on several occasions covering a period of up to two years. The late reflexes were consistently delayed when the side of the lesion was stimulated in both. In one patient (case 6) who pre- sented typical clinical features of the syndrome, the late reflex was within normal limits on three separate examinations (Fig. 1).

Only one patient (case 8) came to necropsy. The reflex study performed while the patient was in coma revealed total absence of the late reflex regardless of the side of stimulation and a delay

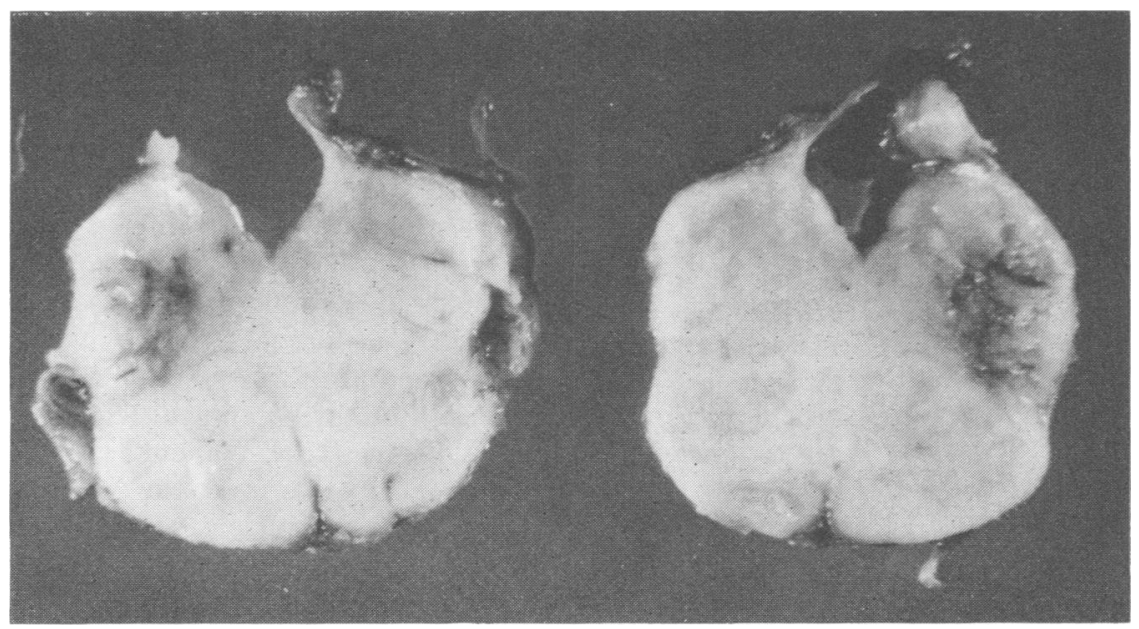

FIG. 3. Case 8. An area of softening with slight haemorrhagic discolouration and a wedge-shaped configuration in the dorsolateral aspect of the medulla on the right, typical of the lateral medullary infarct (by courtesy of Dr.W.F.McCormick, University Hospitals, Iowa City, Iowa). 
of the early reflex on the right. At necropsy, a subacute right lateral medullary infarct (Fig. 3) was noted. Additionally, there was encephalomalacia of both cerebellar hemispheres associated with complete occlusion of the left posterior cerebellar artery. The pons was grossly normal but microscopic examination showed an infarct in the striate portion.

The other patient (case 9) was included in this study as the clinical features, although not typical of the Wallenberg syndrome, suggested a right lateral medullary lesion. The reflex study (Fig. 4) revealed a bilateral delay of the late reflexes on right-sided stimulation in the presence of an essentially normal early reflex on the right.

\section{DISCUSSION}

Alteration of the late reflex may be considered in analogy with the pupillary light reflex. It is thus possible to distinguish, for example, a right afferent delay characterized by a bilateral delay of the late reflexes on right-sided stimulation

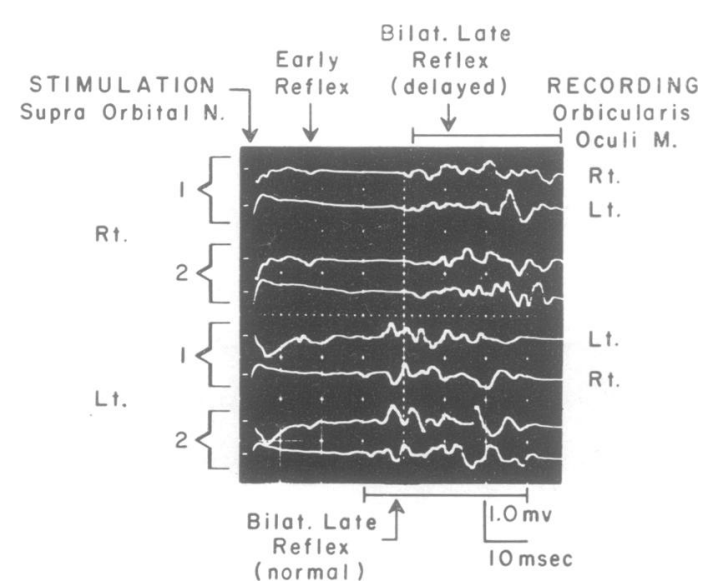

FIG. 4. Case 9. Right lateral medullary lesion. Two successive stimulations (to show consistency) separated by a minimum of 15 seconds on the right (two top pairs) followed by two successive stimulations on the left with the same interval (two bottom pairs). The reflex responses were simultaneously recorded from ipsilateral (upper tracing in each pair) and the contralateral (lower tracing in each pair) orbicularis oculi. Note a unilateral early reflex recorded only in the upper tracing and bilateral late reflexes recorded in both the upper and lower tracings in each pair. The late reflex was consistently delayed bilaterally when the side of the lesion (right) was stimulated and normal bilaterally when the normal side (left) was stimulated. The early reflex (not clearly seen in this amplification) was normal on either side. from a right efferent delay characterized by a $z$ delay of the late reflex on the right regardless of $\stackrel{\odot}{=}$ the side of stimulation.

In the present study, an afferent delay of the late reflex was seen in six out of seven typicalo cases of the Wallenberg syndrome when the side of the lesion was stimulated. This finding is con- $\frac{\overline{2}}{2}$ sistent with the previous observation concerning trigeminal tractotomy in man (Kugelberg, 1952), and in cats (Tokunaga et al., 1958) and $\stackrel{\text { ? }}{9}$ confirms the role of the spinal tract and nucleus? of the trigeminal nerve for the late reflex. In $\frac{\bar{c}}{\mathrm{~s}}$ addition, it may be stated on the basis of the $\overrightarrow{\mathbb{D}}$ present finding that the neurones of the first 0 order responsible for the bilateral late reflexes on ${ }^{\infty}$ unilateral stimulation terminate in the ipsilateral $\vec{\circ}$ spinal nucleus of the trigeminal nerve with no $\overrightarrow{\vec{\omega}}$ significant crossing over to the same structure on the contralateral side. Experimenting in cats, $\overline{\vec{z}}$ Tokunaga et al. (1958) have suggested that the? neurones of the second order responsible for the late reflex cross over, ascend on the contralateral ${ }_{N}^{N}$ side, and then descend diffusely before eventually $N_{\infty}^{N}$ making connection with the facial nuclei bilater-음 ally. No adequate data are yet available in manin this regard.

Alterations in the late reflex demonstrated ing majority of cases with the Wallenberg syndronece contrast sharply with the sparing of this reflex most cases with other medullary lesions. While the late reflex is also affected in some cases with pontine lesions, this change almost invariablyō occurs together with a significantly altered earlyő reflex (Kimura, 1970). In the Wallenberg syn- $\varrho$ drome, on the other hand, the early reflex is $\overrightarrow{0}$ generally within normal limits, although close scrutiny often reveals a borderline delay of the latency on the side of the lesion suggesting some minor pontine dysfunction associated with this syndrome.

A normal late reflex on three separate examinations in one patient (case 6) was exceptional. This patient had only slight hemifacial hypalgesia, although the clinical features were other-o wise typical of the Wallenberg syndrome. It is likely that, in this case, the involvement of the? spinal tract and nucleus of the trigeminal nerve was less than usual and could not be detected by the reflex study. Nearly complete sparing of the $N$ spinal tract is rare but is known to occur in the syndrome (Fisher et al., 1966). Another patient (case 8), seen in coma, escaped detection by thise technique as the late reflexes were totally absent $\stackrel{\Phi}{\Phi}$ with stimulation on either side. In our experience, 
the late reflex is consistently absent or minimal in amplitude in any comatose patient regardless of the cause of coma, presumably due to diffuse suppression of the reticular system.

On the basis of this study it may be concluded that an afferent delay of the late reflex in the presence of a normal or nearly normal early reflex is compatible with a lateral medullary lesion implicating the spinal tract and nucleus of the trigeminal nerve. This finding is commonly seen in the Wallenberg syndrome, although not specific to it as it may be seen in a patient (case 9) whose clinical features are less than adequate to be included in the syndrome. If the neurones of the second order responsible for the late reflex cross over to the other side or are influenced by crossed supranuclear controls in man as has been shown to occur in cats (Tokunaga et al., 1958), an afferent delay or block of the late reflex in the presence of a normal early reflex might well be observed with contralateral mesencephalic, diencephalic or hemispheric lesions.

This work was supported by the Medical Research Council of Canada Operating Grant Number MA4000 and the Multiple Sclerosis Society of Canada, and by the Neurosensory Centre (NSO3354) of the National Institute of Neurological Disease and Stroke of the Department of Health and Education and Welfare of the United States. This work is in part included in the paper presented at the Fourth International Congress of Electromyography on 12 September 1971 in Brussels, Belgium.

\section{REFERENCES}

Bender, L. F., Maynard, F. M., and Hasting, S. V. (1969). The blink reflex as a diagnostic procedure. Archices of Physical Medicine, 50, 27-31.

Fisher, C. M., Karnes, W. E., and Kubik, C. S. (1961). Lateral medullary infarction. The pattern of vascular occlusion. Journal of Neuropathology and Experimental Neurology, 20, 323-379.

Kimura, J. (1970). Alteration of the orbicularis oculi reflex by pontine lesions. Study in multiple sclerosis. Archices of Neurology (Chicago), 22, 156-161.

Kimura, J. (1971). An evaluation of the facial and trigeminal nerves in polyneuropathy: electrodiagnostic study in Charcot-Marie-Tooth disease, Guillain-Barré syndrome, and diabetic neuropathy. Neurology (Minneapolis), 21, 745-752.

Kimura, J., Powers, J. M., and Van Allen, M. W. (1969). Reflex response of orbicularis oculi muscle to supraorbital nerve stimulation. Study in normal subjects and in peripheral facial paresis. Archices of Neurology, 21, 193-199.

Kimura, J., Rodnitzky, R., and Van Allen, M. W. (1970). Electrodiagnostic study of trigeminal nerve. Orbicularis oculi reflex and masseter reflex in trigeminal neuralgia, paratrigeminal syndrome, and other lesions of the trigeminal nerve. Neurology (Minneapolis), 20, 574-583.

Kugelberg, E. (1952). Facial reflexes. Brain, 75, 385-396.

Namerow, N. S., and Etemadi, A. (1970). The orbicularis oculi reflex in multiple sclerosis. Neurology (Minneapolis), 20, 1200-1203.

Rushworth, G. (1962a). Observations on blink reflexes Journal of Neurology, Neurosurgery, and Psychiatry, 25, 93-108.

Rushworth, G. (1962b). The effects of cranial nerve pathology on the blink reflexes. Transactions of the Ophthalmological Society, 82, 549-558.

Shahani, B. (1970). The human blink reflex. Journal of Neurology, Neurosurgery, and Psychiatry, 33, 792-800.

Tokunaga, A., Oka, M., Murao, T., Yokoi, H., Okumura, T., Hirata, T., Miyashita, Y., and Yoshitatsu, S. (1958). An experimental study on facial reflex by evoked electromyography. Medical Journal of Osaka Unitersity, 9, 397411 .

Wilkins, R. H., and Brody, I. A. (1970). Wallenberg's syndrome. Archives of Neurology, 22, 379-382. 